\title{
Functional Photonic Metamaterials
}

\author{
M. Ren ${ }^{1,2}$, J. Y. Ou ${ }^{1}$, B. Jia ${ }^{3}$, E. Plum ${ }^{1}$, J. Zhang ${ }^{1}$, L. Jiang ${ }^{4}$, \\ A. Nikolaenko' ${ }^{1}$ J. J. Xu ${ }^{2}$, M. Gu ${ }^{3}$, K. F. MacDonald ${ }^{1}$, N. I. Zheludev ${ }^{1}$ * \\ ${ }^{1}$ Optoelectronics Research Centre \& Centre for Photonic Metamaterials, \\ University of Southampton, Southampton, SO17 1BJ, UK \\ ${ }^{2}$ Key Laboratory of Weak Light Nonlinear Photonics, Nankai University, Tianjin 300457, P.R. China \\ ${ }^{3}$ Center for Micro-Photonics and CUDOS, Swinburne University of Technology, \\ Melbourne, Victoria 3122, Australia \\ ${ }^{4}$ School of Engineering Sciences, University of Southampton, Southampton, SO17 1BJ, UK \\ *niz@orc.soton.ac.uk; www.nanophotonics.org.uk
}

\begin{abstract}
We review our recent results in the development of nanostructured photonic metamaterials which provide unprecedented levels of active (nonlinear, switchable, tunable) control over light on the sub-wavelength scale.
\end{abstract}

Photonic metamaterials were conceived originally as a paradigm for achieving new and unusual passive electromagnetic properties (such as negative refraction and artificial magnetism) via the subwavelength structuring. However, they have evolved rapidly to form the basis of active devices wherein the structuring of plasmonic metals and hybridization with active media delivers novel and/or enhanced nonlinear, switchable and tunable responses that can be harnessed to control light on the nanoscale.

Here we describe recent advances in the experimental development of functional photonic metamaterials, including demonstrations of some of the largest and fastest engineered optical

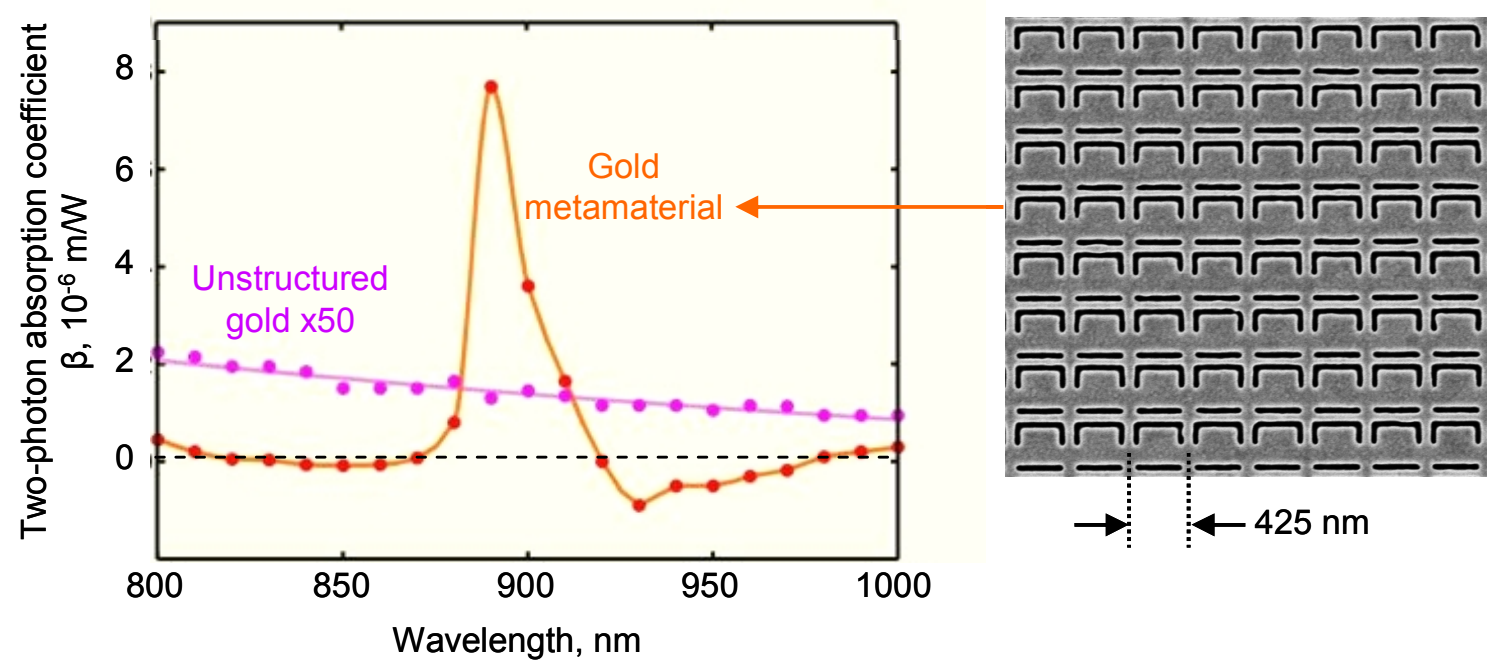

Fig. 1: Two-photon absorption coefficient of a gold metamaterial (shown right: $50 \mathrm{~nm}$ gold film on a quartz substrate patterned by focused ion beam milling with an array of asymmetric split ring resonators) compared to that of an unstructured gold film (multiplied by a factor of 50). The nonlinearity of the nanostructured metal is clearly seen at peak intensities of just a few $\mathrm{GW} / \mathrm{cm}^{2}$ and shows a dramatic resonance coinciding with the 'closed mode' linear absorption peak of the metamaterial at the plasmonic where $\beta=7.7 \times 10^{-6}(\sim 300 \times$ the value for unstructured gold). 
nonlinearities reported to date: it is found that plasmon-exciton coupling strongly enhances the sub-500 fs nonlinear response of semiconducting carbon nanotubes in a hybrid metamaterial system; and that metamaterial structuring can dramatically enhance the sub-100 fs two-photon nonlinearity of gold itself by a factor of 300 (Fig. 1).

Switchable and continuously tunable systems are also discussed, including mechanically reconfigurable electrostatically-driven photonic metamaterials (Fig. 2). In such structures the positioning of nanoscale plasmonic resonators on reconfigurable supports allows for inter-resonator interactions to be controlled, leading to tuning of their collective electromagnetic properties.

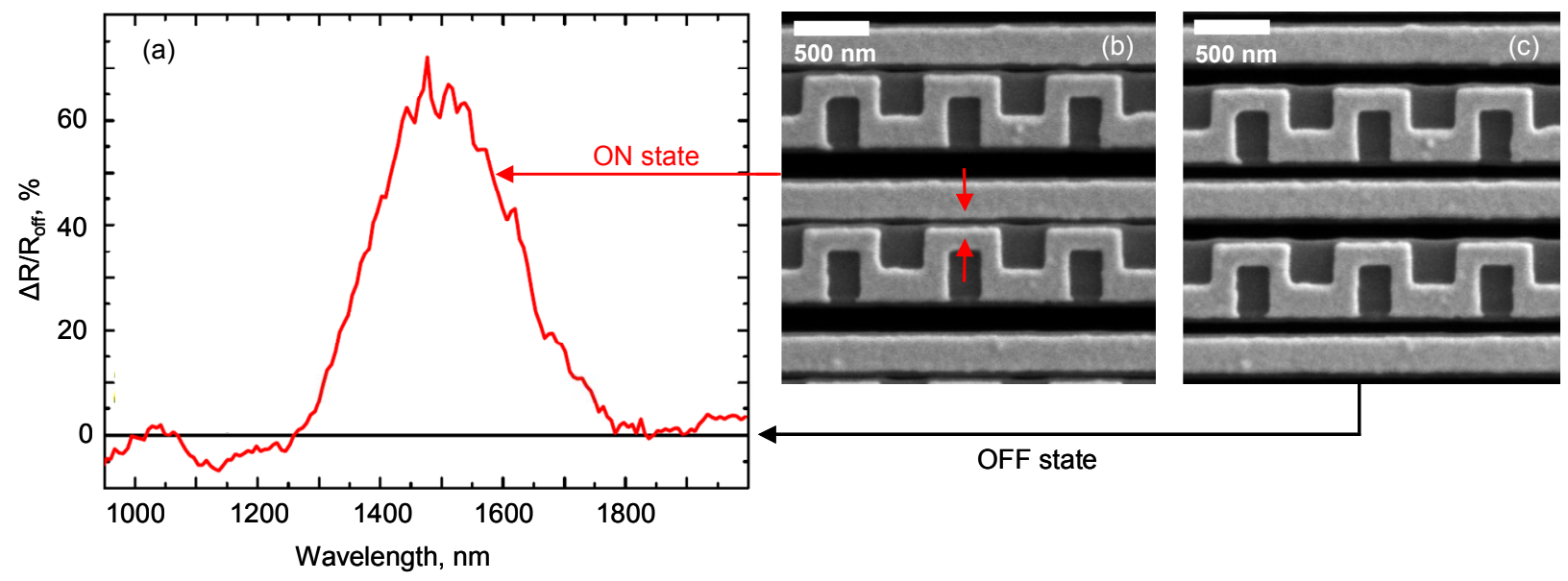

Fig. 2: Reflection contrast (a) between 'on' (b) and 'off' (c) states of an electrostatically-driven reconfigurable metamaterial. The structure (manufactured by focused ion beam milling on a $50 \mathrm{~nm} \mathrm{Si}_{3} \mathrm{~N}_{4}$ membrane coated with a $50 \mathrm{~nm}$ gold film) comprises $35 \mu \mathrm{m}$ long bridges alternately patterned with 'fishscale' plasmonic resonators and straight gold wires. Connecting bridges to a voltage source such that alternate pairs are at opposite polarity provides electrostatic control over bride spacing. At a threshold of $5.7 \mathrm{~V}$ every other gap in the structure is closed. This switch to the 'on' state changes the coupling between neighbouring plasmonic elements in the array and brings about a dramatic change in the optical properties of the metamaterial: a $72 \%$ increase in reflectivity. 